\title{
INTERACTION OF A CAVITATION BUBBLE WITH AN ANTIBUBBLE
}

\author{
BAI LIXIN ${ }^{1}$, XU WEILIN ${ }^{2}$, YAN JIUCHUN ${ }^{3} \&$ ZENG ZHIJIE $^{1}$ \\ ${ }^{1}$ State Key Laboratory of Acoustics, Institute of Acoustics, Chinese Academy of Sciences, China \\ ${ }^{2}$ State Key Laboratory of Hydraulics and Mountain River Engineering, Sichuan University, China \\ ${ }^{3}$ State Key Laboratory of Advanced Welding Production Technology, Harbin Institute of Technology, China
}

\begin{abstract}
An antibubble is the opposite of a bubble: a thin spherical gas shell containing liquid inside and surrounded by liquid outside. Although antibubbles are rarely used in industry, they do have some potential applications, such as cleaning, lubrication, filtration and material transport. The dynamic behaviour of antibubbles and spark-induced cavitation bubbles is experimentally studied by using highspeed photography. It is found that when spark discharges occur between the electrodes and the cavitation bubble begin to expand, the antibubble splits into upper and lower parts. The upper parts with more gas produces annular liquid jet under the action of shock wave, and split into two bubbles in the next few cycles under the action of inertial force. The lower parts are composed of semi-spherical shell-like gas film. When the compression wave arrives, the gas film becomes thinner and contacts between the two sides of the gas film appear at some points. When the expansion wave arrives, the gas film becomes thicker and the contact point becomes larger to form holes. As the holes become larger and the gas film shrinks, many scattered microbubbles are formed. The secondary shock wave caused by the collapse of the cavitation bubble which occur a few milliseconds after the first shock wave (spark discharge) was observed by schlieren photography. The physical mechanism of interaction between cavitation bubble and antibubble is analysed.
\end{abstract}

Keywords: cavitation bubble, antibubble, film, shock wave, high-speed photography.

\section{INTRODUCTION}

Cavitation refers to the formation and subsequent dynamic life of bubbles in liquids subjected to a sufficiently low pressure [1]. Extreme high temperature and pressure could be generated inside the cavitation bubble during the collapse, and shock waves and microjets could be generated in the liquid outside the cavitation bubbles. Cavitation can concentrate the diffuse energy to produce many effects with many applications. Applications of cavitation span many industrial sectors, from ultrasonic brazing, through ultrasonic lithotripsy, sonochemistry, ultrasonic cleaning, and wastewater treatment, to jet cutting. These applications of cavitation occur through the interaction between cavitation bubbles and other objects. Many investigation have been carried out. Lindau and Lauterborn [2], Shaw et al. [3], Brujan et al. [4] and Tomita et al. [5] investigated the interaction between a cavitation bubble and a rigid boundary. Robinson et al. [6] analysed the motion of two cavitation bubbles in a line perpendicular to the free surface. Bai et al. [7] investigated the interaction of an air bubble (isolated in water or attached to a boundary) with shock waves induced by electric sparks. Kodama et al. [8] investigated the interaction of a cavitation bubble with two air bubbles attached to a boundary. Poulain et al. [9] investigated the interaction of a cavitation bubble with a distant and small tethered spherical particle. Until recently, the interaction of a cavitation bubble with a gas film (antibubble) has not been studied.

An antibubble is the opposite of a soap bubble: a thin spherical gas shell containing liquid inside and surrounded by liquid outside. Although antibubbles are rarely used in industry, they do have some potential applications, such as cleaning, lubrication, filtration and material transport. Many investigation have been carried out on formation [10]-[14], aging [15]-[18], 
collapse [19], [20] and stabilization [21]-[23] of antibubbles. However, no investigation on the interaction between antibubbles and other fluid objects has been carried out.

In this paper, the interaction of a cavitation bubble with an antibubble is experimentally studied by using high-speed photography. The dynamic behaviour of antibubbles and sparkinduced cavitation bubbles is analysed. The present work is, from the authors' knowledge, the first analysis on the interaction of a cavitation bubble with an antibubble.

\section{EXPERIMENT}

The experimental setup consists of a cavitation bubble generation system, an antibubble generation system, and imaging and illumination devices (Fig. 1). Cavitation bubbles are generated by using high-voltage spark discharge. The discharge time is of special importance, and is accurately controlled. Fine copper wires are used as electrodes in the experiment. The dripping device can produce jets of different velocities and droplets of different sizes. A rectangular plexiglass container $(220 \mathrm{~mm} \times 150 \mathrm{~mm} \times 170 \mathrm{~mm})$ is used to hold a mixture of tap water and linear alkylbenzenesulfonate (LAS) (about 10 times the critical micellar concentration $(2.2 \mathrm{mM})$. The behaviour of cavitation bubbles and antibubbles is recorded with a high-speed camera (Photron Fastcam SA-1, Photron Ltd., Japan) equipped with two long distance microscopes (Zoom 6000, Navitar, USA; LM50JCM, Kowa, Japan) respectively. The frames are illuminated with PI-LUMINOR high-light LED lamp (150W), Cree XHP70 white high power LED, HALOGEN lamp (2600W). In the experiment, the liquid in the dripping device is the same as that in the rectangular glass tank. The gas in the antibubble film is the air in the environment (temperature: $20^{\circ} \mathrm{C}$, pressure: $1 \mathrm{~atm}$ ).

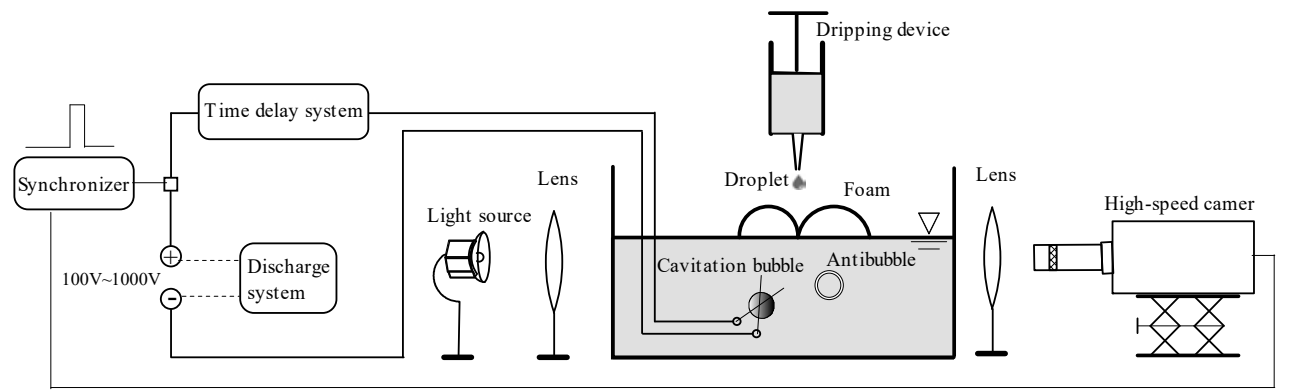

Figure 1: Experimental setup.

\section{RESULTS AND DISCUSSION}

Using fine copper wire as electrodes can eliminate the influence of electrodes as far as possible and ensure the sphericity of the cavitation bubble. The growth and collapse of a spark-induced cavitation bubble is shown in Fig. 2. It is found in the experiment that the primary shock wave produced by the instantaneous energy release of spark discharge is difficult to photograph because of the strong light produced by spark (as shown the schematic diagram in Fig. 2). The secondary shock wave produced by the collapse of cavitation bubble is visualized clearly by schlieren photography (as shown the subfigure on the right in Fig. 2). The primary shock wave and the secondary shock wave will affect the deformation of antibubbles. 
It is found in the experiment that antibubbles will split horizontally into two parts (upper part and lower part) during the growth of the cavitation bubble (Fig. 3). The splitting line is always near the top of the antibubble and always horizontal. Through many experiments, it is found that there is a stable phase relationship between the cavitation bubble and the antibubble (Fig. 4). A large number of experiments show that the antibubble splitting characteristics are independent of azimuth (Fig. 5). Experiments show that if the primary shock wave is too strong, the antibubble may completely break into microbubbles without splitting (Fig. 6). In this paper, only moderate- and low-intensity shock waves are discussed.

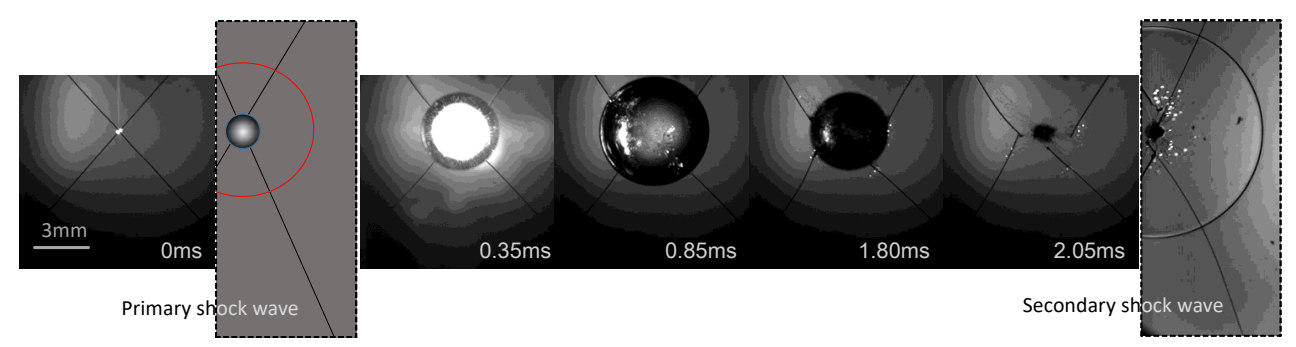

Figure 2: The growth and collapse of a spark-induced cavitation bubble.

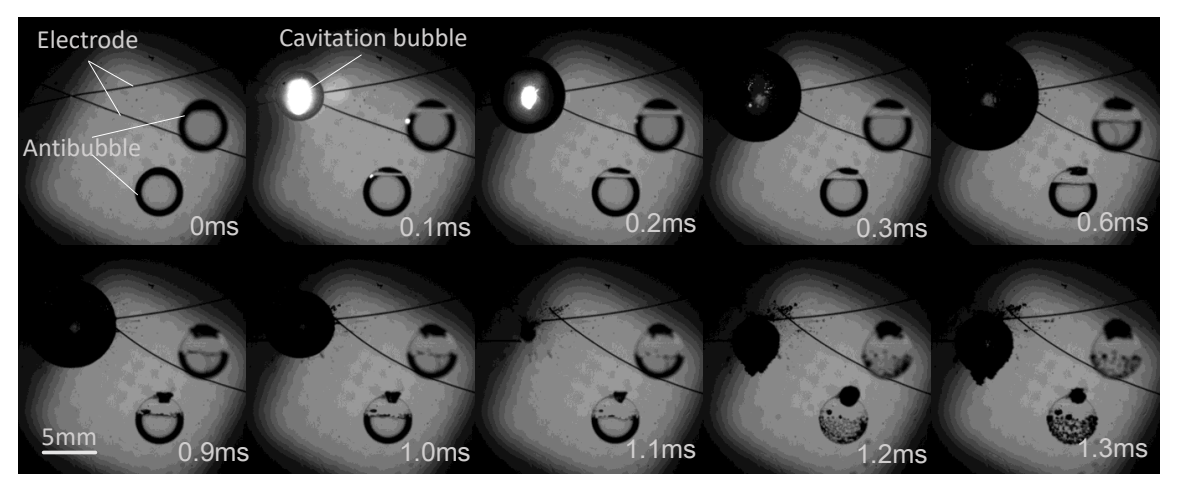

Figure 3: Interaction of a cavitation bubble with two antibubbles (medium-intensity shock wave).

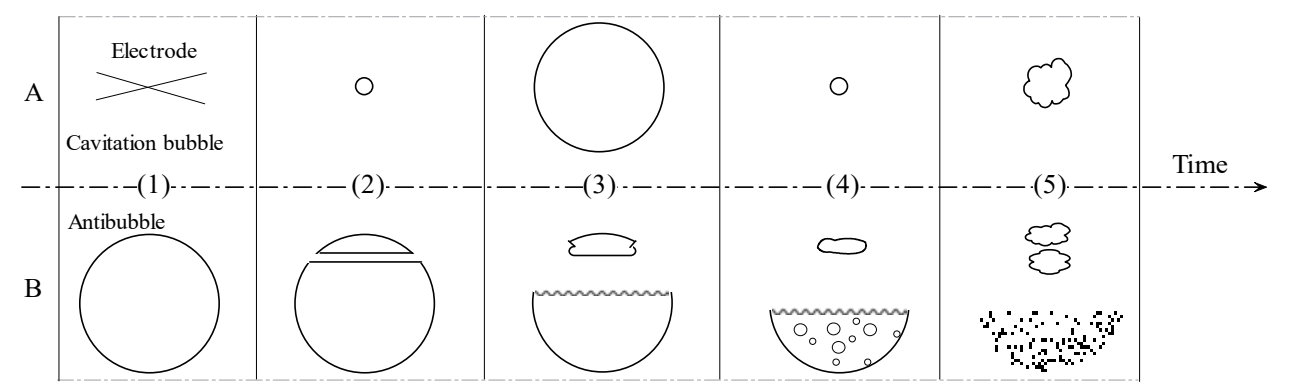

Figure 4: Schematic diagram of phase relation between cavitation bubble deformation and antibubble deformation. 


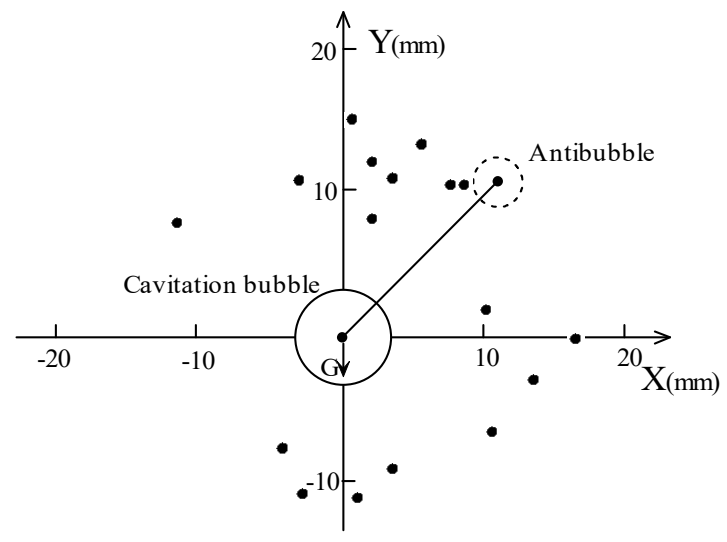

Figure 5: Relative positions of cavitation bubble and antibubble in the experiments.

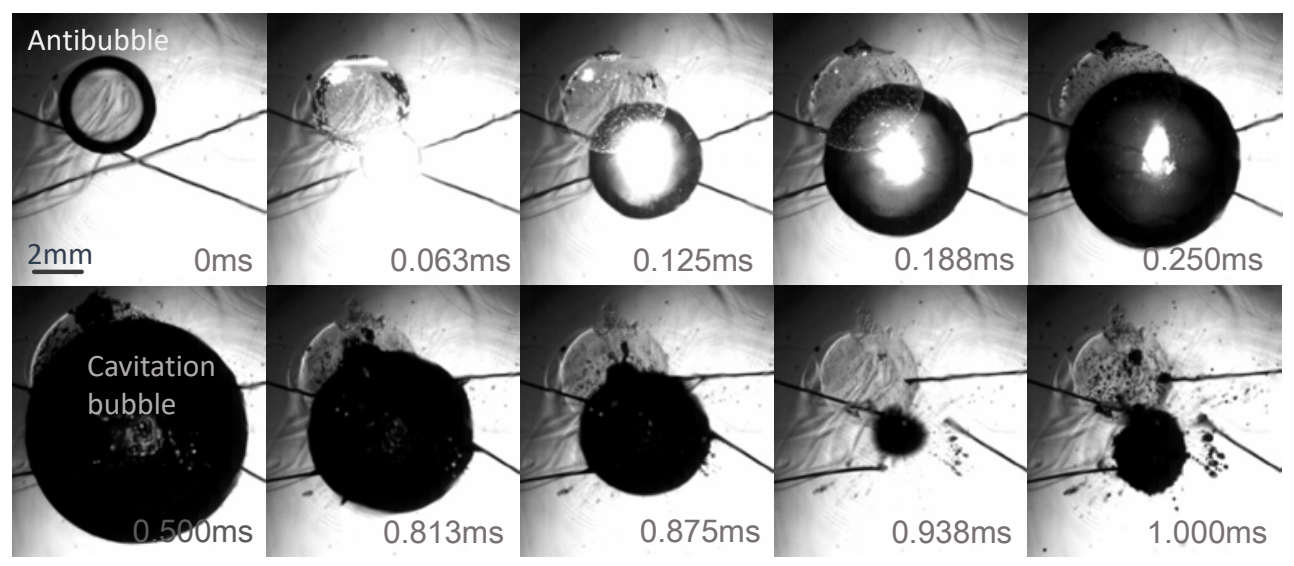

Figure 6: Interaction of a cavitation bubble with a antibubble (high-intensity shock wave).

The detailed process of antibubble deformation can be seen from Fig. 7. The lower edge of the upper part of the antibubble shrinks inward and produces a liquid jet (Fig. 7(a) (13)). When the secondary shock wave arrives, the upper part of the antibubble appears as a flat disc (Fig. 7(a) (25) and Fig. 7(b) $(\tau=0.8033 \mathrm{~ms})$ ). Then, the upper part of the antibubble splits vertically into two air bubbles (Fig. 7(a) (36) and Fig. 7(b) $(\tau=1.2 \mathrm{~ms}$ )). The upper edge of the lower part of the antibubble shrinks downward until the secondary shock wave arrives (Fig. 7(a) (1)-(24) and Fig. 7(b) $(\tau=0-0.8 \mathrm{~ms})$ ). There are some holes in the gas film (Fig. 7(a) (25) and Fig. 7(b) $(\tau=0.8033 \mathrm{~ms}))$. Then the holes became larger and the gas film fragmented into many microbubbles.

Because the gas density is smaller than that of water, under the action of gravity and buoyancy, the gas in the lower part of the antibubble film will gather up to the upper part, so the upper gas film is thicker than the lower one (Fig. 8 (1)). When the primary shock wave arrives, the gas film in the antibubble is compressed. Because the upper part of antibubble contains more gas, so the upper part shrinks more obviously. The suction effect of the upper 


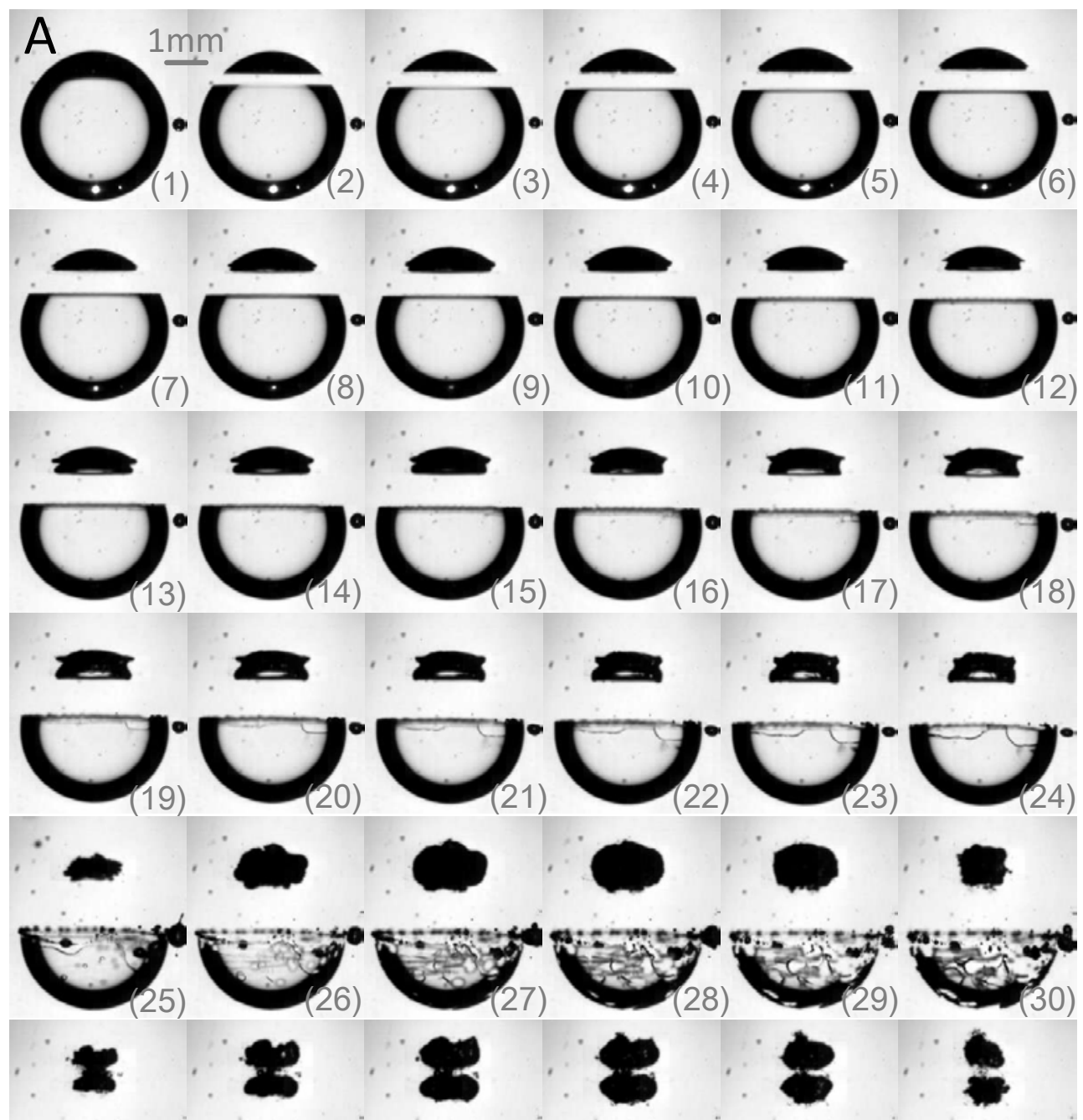

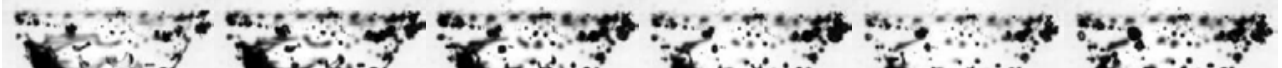

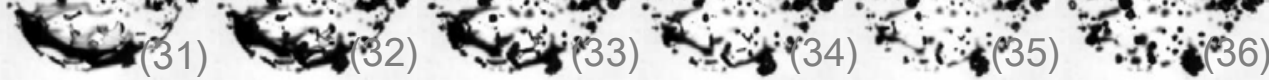

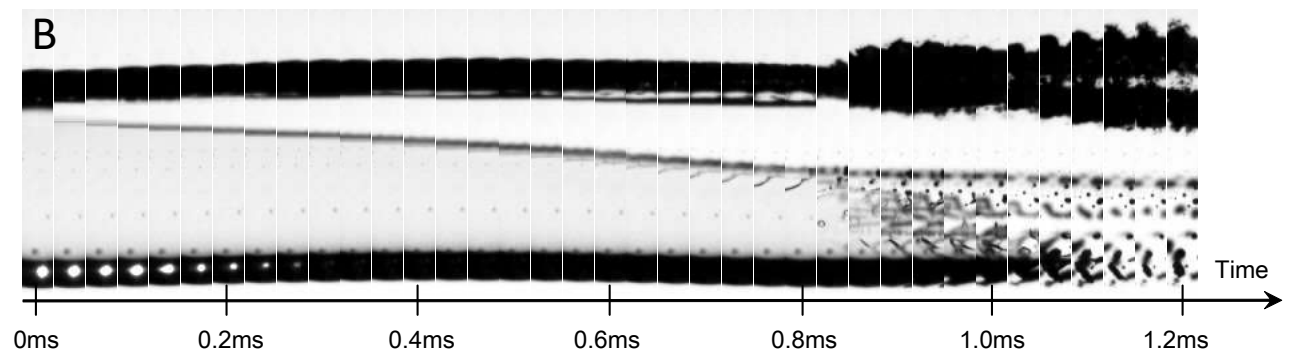

Figure 7: Deformation of antibubble. (a) High-speed photos (Frame rate $30000 \mathrm{fps,}$ exposure time 1/184000 s); and (b) Streak plot of antibubble deformation. 
gas causes the film to break somewhere. The fracture location is related to the thickness (d) of the gas film at that location and the distance (L) from the center of gravity of gas film to that location. The points that meets this condition will form a horizontal circle. After disconnecting from the lower part, the lower edge of the upper part of antibubble shrinks inward under the action of surface tension, and form a liquid jet (Fig. 8 (3)). Because the direction of the jet is closer to the tangent direction of the arc, the jet does not cause the splitting of the upper part of antibubble. When the secondary shock wave arrives, the upper part of the antibubble appears as a flat disc (Fig. $8(5,6)$ ). After the shock wave and its subsequent negative pressure pass, the flat disc air bubble shrinks inward and forms a circular jet under the action of surface tension. The circular jet divides the air bubbles into two (Fig. $8(7,8)$ ).

Although the gas film of lower part of antibubble is very thin (submicron to micron), its impact resistance is stronger than we expected. The primary shock wave did not cause any damage to the gas film. It is well known that the secondary shock wave is stronger than the primary shock wave. The secondary shock waves cause the two interfaces of the gas film to stick together at some point (Fig. 9 (2) and Fig. 10 (2)). The stronger the shock wave, the

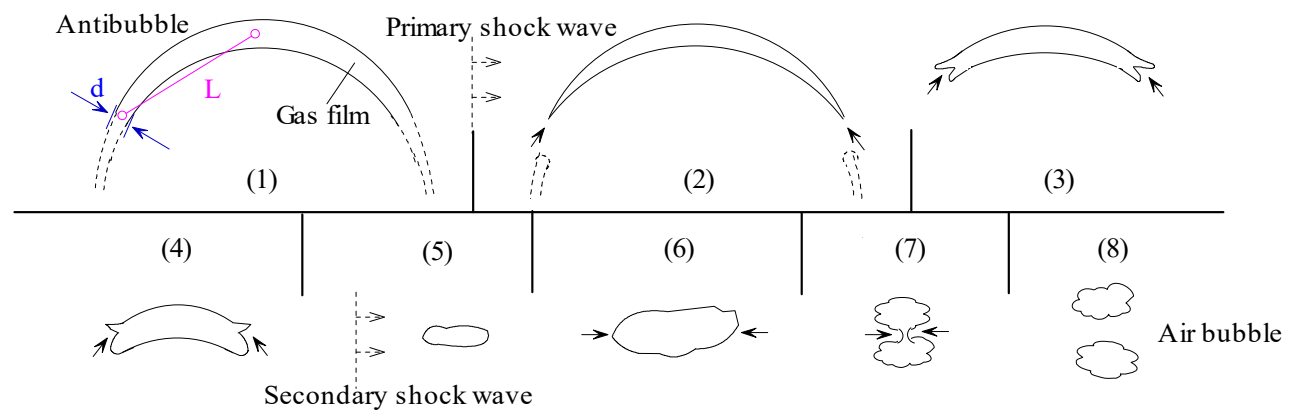

Figure 8: Schematic diagram of deformation of upper part of antibubble.

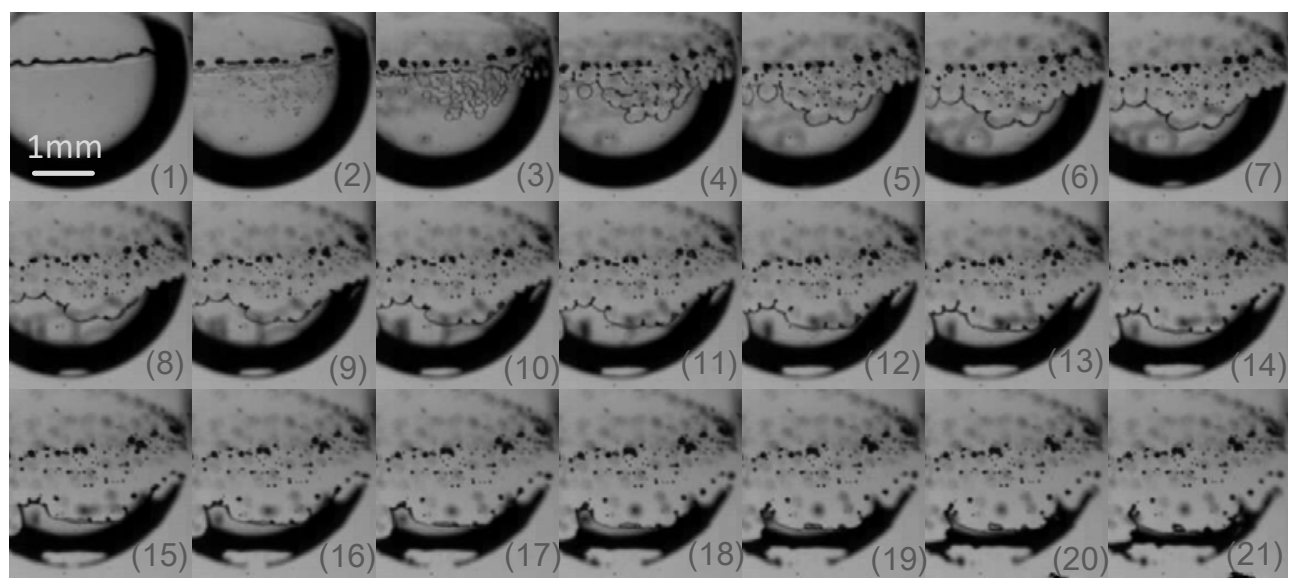

Figure 9: Breakage of gas film in lower part of antibubble (Frame rate $40000 \mathrm{fps}$, exposure time $1 / 307000 \mathrm{~s})$. 


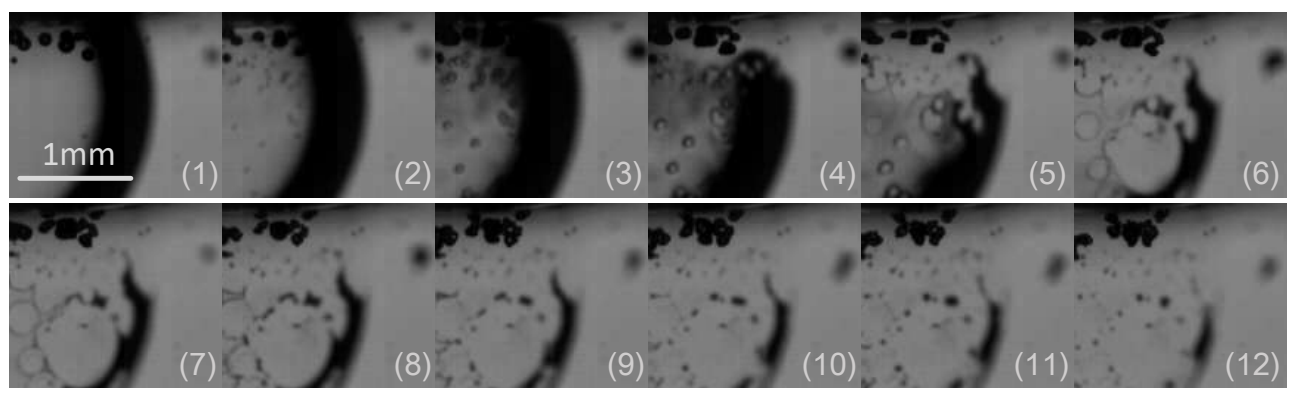

Figure 10: Details of gas film rupture (Frame rate 100000 fps. Exposure time 1/297000 s).

more points of contact. Under the action of surface tension, the contact points become holes. The gas gathers around the hole (thicker than the gas film), forming a ring-shaped gas bubble connected with the gas film (Fig. 9 (3) and Fig. 10 (3-5)). As the holes become larger, they connect with each other and break to form many microbubbles.

The total reflection caused by the gas-liquid interface on both sides of the gas film makes the antibubble show a thick black edge on the outer edge in the transmission light. Under the impact of secondary shock wave, the inner edge of the black edge blurs into gray (Fig. 11(a) (2) and Fig. 11(b) (2)). This indicates that a small amount of light passes through the original total reflection region. The contact point of the film is probably the channel through which the small amount of light may pass (Fig. 11(b) (3)).
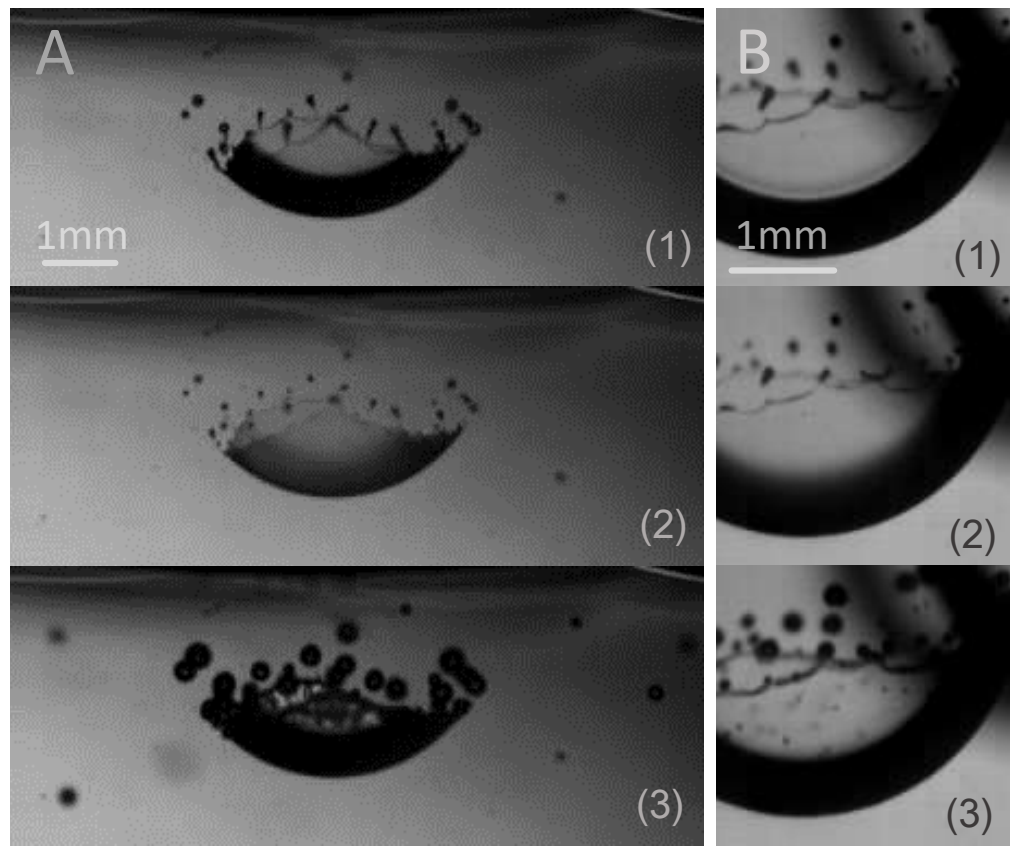

Figure 11: Black edge caused by total reflection (Frame rate 100000 fps, exposure time $1 / 297000 \mathrm{~s})$. 


\section{SUMMARY AND CONCLUSIONS}

The interaction of a spark-induced cavitation bubble with an antibubble is experimentally investigated by using high-speed photography. It is found that there is a stable phase relationship between the cavitation bubble deformation and the antibubble deformation. Antibubbles are always divided horizontally into two parts, independent of azimuth. The suction effect of the upper gas causes the gas film to break. The fracture location is related to the thickness of the gas film at that location and the distance from the center of gravity of gas film to that location. The formation of the liquid jet in the upper part of the antibubble and the splitting of the disc-shaped air bubble are all related to the surface tension. The secondary shock waves cause the two interfaces of the gas film in the lower part of antibubbles to stick together at some point. These defects eventually lead to partial loss of total reflection and the fragmentation of the gas film.

\section{ACKNOWLEDGEMENT}

This work was supported by the National Natural Science Foundation of China (No. 11874062, No. 11674350).

\section{REFERENCES}

[1] Bai, L., Xu, W., Tian, Z. \& Li, N., A high-speed photographic study of ultrasonic cavitation near rigid boundary. Journal of Hydrodynamics, 20, pp. 637-644, 2008.

[2] Lindau, O. \& Lauterborn, W., Cinematographic observation of the collapse and rebound of a laser-produced cavitation bubble near a wall. Journal of Fluid Mechanics, 479, pp. 327-348, 2003.

[3] Shaw, S.J., Schiffers, W.P. \& Emmony, D.C., Experimental observations of the stress experienced by a solid surface when a laser-created bubble oscillates in its vicinity. Journal of the Acoustical Society of America, 110, pp. 1822-1827, 2001.

[4] Brujan, E.A., Keen, G.S., Vogel, A. \& Blake, J.R., The final stage of the collapse of a cavitation bubble close to a rigid boundary. Physics of Fluids, 14, pp. 85-92, 2002.

[5] Tomita, Y. et al., Growth and collapse of cavitation bubbles near a curved rigid boundary. Journal of Fluid Mechanics, 466, pp. 259-283, 2002.

[6] Robinson, P.B., Blake, J.R., Kodama, T., Shima, A. \& Tomita, Y., Interaction of cavitation bubbles with a free surface. Journal of Applied Physics, 89, pp. 8225-8237, 2001.

[7] Bai, L., Xu, W., Li, C. \& Gao, Y., The counter jet formation in an air bubble induced by the impact of shock waves. Journal of Hydrodynamics, 23(5), pp. 562-569, 2011.

[8] Kodama, T., Takayama, K. \& Nagayasu, N., The dynamics of two air bubbles loaded by an underwater shock wave. Journal of Applied Physics, 80, pp. 5587-5592, 1996.

[9] Poulain, S., Guenoun, G., Gart, S., Crowe, W. \& Jung, S., Particle motion induced by bubble cavitation. Physical Review Letters, 114(21), 214501, 2015.

[10] Bai, L., Xu, W., Wu, P., Lin, W. \& Li, C., Formation of antibubbles and multilayer antibubbles. Colloids and Surfaces A: Physicochemical and Engineering Aspects, 509(20), pp. 334-340, 2016.

[11] Ganan-Calvo, M. \& Gordillo, J.M., Perfectly monodisperse microbubbling by capillary flow focusing. Physical Review Letters, 87, 274501, 2001.

[12] Tufaile, A. \& Sartorelli, J.C., Bubble and spherical air shell formation dynamics. Physical Review E, 66, 056204, 2002.

[13] Kim, P.G. \& Stone, A.H., Dynamics of the formation of antibubbles. Europhysics Letters, 83, 54001, 2008. 
[14] Postema, M., Ten Cate, F.J., Schmitz, G., De Jong, N. \& Van Wamel, A., Generation of a droplet inside a microbubble with the aid of an ultrasound contrast agent: First result. Letters in Drug Design and Discovery, 4, pp. 74-77, 2007.

[15] Dorbolo, S., Reyssat, E., Vandewalle, N. \& Quéré, D., Aging of an antibubble. Europhysics Letters, 69, pp. 966-970, 2005.

[16] Dorbolo, S. et al., Antibubble lifetime: Influence of the bulk viscosity and of the surface modulus of the mixture. Colloids and Surfaces A, 365, pp. 43-45, 2010.

[17] Scheid, B., Dorbolo, S., Arriaga, L.R. \& Rio, E., The drainage of an air film with viscous interfaces. Physical Review Letters, 109, 264502, 2012.

[18] Scheid, B., Zawala, J. \& Dorbolo, S., Gas dissolution in antibubble dynamics. Soft Matter, 10, pp. 7096-7102, 2014.

[19] Sob'yanin, D.N., Theory of the antibubble collapse. Physical Review Letters, 114, 104501, 2015.

[20] Zou, J., Ji, C., Yuan, B.G., Ruan, X.D. \& Fu, X., Collapse of an antibubble. Physical Review E, 87, 061002(R), 2013.

[21] Dorbolo, S., Caps, H. \& Vandewalle, N., Fluid instabilities in the birth and death of antibubbles. New Journal of Physics, 5, pp. 161.1-161.9, 2003.

[22] Kim, P.G. \& Vogel, J., Antibubbles: Factors that affect their stability. Colloids and Surfaces A, 289, pp. 237-244, 2006.

[23] Poortinga, A.T., Long-lived antibubbles: Stable antibubbles through Pickering stabilization. Langmuir, 27, pp. 2138-2141, 2011. 